Carsten Tautorat, Frank Kamke, Sylvia Pfensig, Stefan Siewert, Klaus-Peter Schmitz, Niels Grabow, Wolfram Schmidt*

\title{
Multi-channel impedance analyzer for auto- mated testing of networks and biomaterials
}

\begin{abstract}
Impedance spectroscopy represents a basic operating principle for biomedical sensors, bioimpedance spectroscopy, electrochemical analyses and for characterization of functional biomaterials. For automated long-term investigations, an impedance analyzer for multi-channel testing of up to eight passive two-pole networks is presented in this paper. Its operating system is application-specific adapted to the required test functionalities and measuring ranges. Measurements are based on a commercially available integrated impedance converter circuit. Our current analyzer setup is capable of measuring impedance values from $50 \mathrm{k} \Omega$ up to $10 \mathrm{M} \Omega$ with automated range selection for most accurate results. The impedance under test is excited with a single frequency of $1 \mathrm{kHz}$. An impedance accuracy of $1.5 \%$ was determined in reference measurements. The presented impedance analyzer is a low cost system ready for use particularly in long-term characterization of dielectric networks, such as material properties, with multiple samples.
\end{abstract}

Keywords: impedance, dielectric, automated, analyzer

https://doi.org/10.1515/cdbme-2020-3107

\section{Introduction}

Impedance spectroscopy represents a standard method used for the determination of electrical components and circuit networks. Apart from electrical engineering, impedance technique has a wide range of applications, e.g. in the field of biomedical engineering as basic operating principle for biomedical sensors, bioimpedance spectroscopy, electrochemical analyses and for characterization of functional biomateri-

\footnotetext{
${ }^{*}$ Corresponding author: Wolfram Schmidt: Institute for Biomedical Engineering, Rostock University Medical Center, Friedrich-Barnewitz-Str. 4, 18119 Rostock, Germany, e-mail: wolfram.schmidt@uni-rostock.de

Carsten Tautorat, Klaus-Peter Schmitz, Niels Grabow: Institute for Biomedical Engineering, Rostock University Medical Center, Friedrich-Barnewitz-Str. 4, 18119 Rostock, Germany Frank Kamke, Sylvia Pfensig, Stefan Siewert, Klaus-Peter Schmitz: Institute for ImplantTechnology and Biomaterials e.V., Friedrich-Barnewitz-Str. 4, 18119 Rostock, Germany
}

als. Impedance analyzers are also used to measure the frequency dependent impedance and phase of these special electrical networks, exhibiting dielectric behavior. Based on equivalent circuit models, impedance parameters, such as conductivity and capacitance, are calculated from measured impedance values.

Our developed multi-channel impedance analyzer allows for monitoring and evaluating long-term effects of dielectric networks or materials. Up to eight passive two-pole networks can be measured successively with short delay times, quasi in parallel. This large number of channels allows us increasing throughput as well as varying different application-specific test parameters at the same time. The impedance of a network is determined by excitation with a known frequency. For efficient long-term investigations, we reduce impedance data recording to a fixed single frequency value. This characteristic frequency point is determined from previous application-specific spectroscopy tests with frequency sweeps. The impedance under test (IUT) is currently excited with a frequency of $1 \mathrm{kHz}$.

\section{Material and methods}

\subsection{Impedance analyzer}

The multi-channel impedance analyzer mainly consists of up to eight application-specific configured headstages connected to a control unit, see Figure 1.

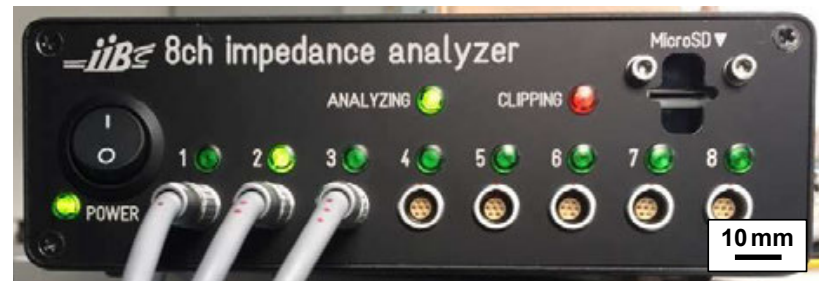

Figure 1: Multi-channel impedance analyzer (front view) with three connected headstages. Headstage \#2 is operated.

Each headstage includes high-sensitive front-end electronics for wide range impedance measurements from $50 \mathrm{k} \Omega$ to $10 \mathrm{M} \Omega$, located as close as possible to the IUT for optimal signal-to-noise ratio (SNR). The IUT is excited with a sinusoidal $1 \mathrm{kHz}$ excitation signal. Calibration settings, excitation 
voltage amplitude as well as IUT signal amplification are automatically selected by the control unit, for most accurate results. Headstages and control unit are mixed signal designs that consist of sophisticated analog and digital electronics.

\subsection{Headstage}

Impedance measurements are based on the commercially available integrated impedance converter circuit AD5933 (Analog Devices Inc., USA) with integrated excitation voltage generator and signal processing, advanced with external circuitry including calibration, IUT signal amplification and range selection, see Figure 2 A [1]. The IUT is connected to the buffered excitation voltage and to the input stage of a transimpedance amplifier (TIA) [2,3]. The TIA converts the sensitive current response signal from the IUT into amplified voltage for signal processing. An integrated digital signal processor (DSP) processes the sampled response signal with discrete Fourier transform to generate the real and imaginary components [2]. After measuring, the headstage sends a digital representation of the real and imaginary part to the control unit for calculating the actual impedance values. To achieve best flexibility, all headstages are star-shaped connected to the digital control unit via $2 \mathrm{~m}$ multi-core cables, as suggested in Figure 3 A.

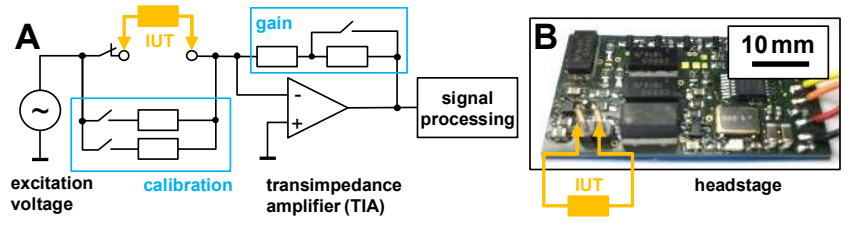

Figure 2: Headstage circuitry for impedance measurements. A: Simplified circuit diagram. B: Fully equipped headstage PCB.

Headstage electronics are single-sided assembled on a smallsize printed circuit board (PCB) without housing for simple application integration, see Figure 2 B. We use rail-to-rail amplifiers featuring very low offset voltage, low input bias, low input noise, and wide signal bandwidth for maximum TIA gain as well as for excitation voltage buffering, required for increasing SNR. The measuring sequence always provides maximum allowed response signals, due to the out-ofrange detection based on two TIA output voltage thresholds. Four amplitude levels are software selectable for excitation, see Table 1. For applications, such as electrochemical analyses, the excitation voltage may have to be limited due to electrolysis or other undesirable electrode processes. In addition, care must be taken, because the IUT is biased to $2.5 \mathrm{~V}$, due to virtual grounding. The programmable two-stage TIA gain is configured with a reed relay in the feedback, see Figure 2. The resulting TIA gain factor is determined during calibration. Therefore, no precision resistors are required in this circuit part. The active TIA output signal is multiplexed to a BNC connection on the rear panel of the control unit for connecting to a data logger or an oscilloscope. An EEPROM is used to store the headstage configuration and calibration settings, as listed in Table 1. TIA gain and calibration are currently optimized for application-specific IUT near $0.5 \mathrm{M} \Omega$ and $2 \mathrm{M} \Omega$. The control unit reads this information prior to each measurement, so that it is possible to operate in parallel with differently configured headstages.

Table 1: Range selection, optimized for $0.5 \mathrm{M} \Omega$ and $2 \mathrm{M} \Omega$. Out-ofrange detection: TIA output voltage thresholds: $<0.2 \mathrm{~V}$ and $>4.8 \mathrm{~V}$.

\begin{tabular}{|c|c|c|c|c|c|c|c|c|}
\hline Range selection & 1 & 2 & 3 & 4 & 5 & 6 & 7 & 8 \\
\hline $\begin{array}{l}\text { Excitation } \\
\text { voltage }(p-p)[V]\end{array}$ & 3 & 1.47 & 0.58 & 0.3 & 3 & 1.47 & 0.58 & 0.3 \\
\hline \multicolumn{9}{|c|}{ Application-specific optimized for IUT near $0.5 \mathrm{M} \Omega$ and $2 \mathrm{M} \Omega$} \\
\hline TIA gain $[\mathrm{M} \Omega]$ & \multicolumn{4}{|c|}{2.02} & \multicolumn{4}{|c|}{0.51} \\
\hline $\begin{array}{l}\text { Calibration } \\
\text { resistor [M } \Omega]\end{array}$ & \multicolumn{4}{|c|}{2} & \multicolumn{4}{|c|}{0.51} \\
\hline Upper limit $[\mathrm{M} \Omega]$ & 10 & $<1.32$ & $<0.65$ & $<0.26$ & 0.5 & $<0.33$ & $<0.16$ & $<0.06$ \\
\hline Lower limit $[\mathrm{M} \Omega]$ & 1.32 & 20.65 & 0.26 & 0.13 & 0.33 & 0.16 & 0.06 & 0.03 \\
\hline
\end{tabular}

\subsection{Control unit}

Operating system and measuring sequence are controlled by an Arduino Uno Rev3 microprocessor development board (Arduino LLC, USA), implemented on a developed PCB in the control unit. $\mathrm{I}^{2} \mathrm{C}$ bus communication is used for interfacing almost all peripheral circuits to the development board, including port expander, real-time clock, microSD memory card module and all headstages. The control unit is mounted in a shielded metal case, providing electrical connectors for eight headstages on the front panel, see Figure 1. Nearby LEDs indicate the currently operated headstage. A connection monitor recognizes open headstage channels. These channels are skipped in the measuring sequence and a comment is stored in the impedance data. On the rear panel, the control unit is equipped with an USB interface for application-specific adaption of the operating system and measuring sequence. Starting with the first headstage (\#1) the measuring sequence is triggered by using an external digital start signal, see Figure 3B. We are using a programmable interval timer for generating a periodically repeated start signal for long-term measurements. In addition, the timer features a button for manual triggering. The unknown IUT from all connected headstages are successively 
determined, comprising initialization, calibration, impedance measuring and if necessary the adaption of excitation voltage, TIA gain or calibration resistor, due to range selection and out-of-range detection, see Figure 3 B.
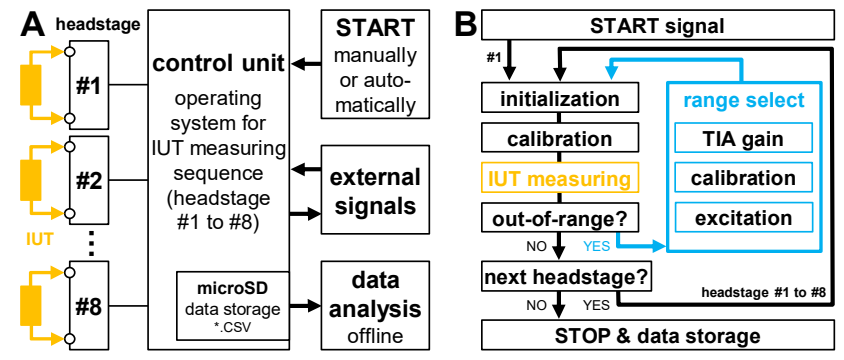

Figure 3: Multi-channel impedance measurements. A: Simplified bloc diagram of the impedance analyzer. B: Simplified flow chart of the measuring sequence.

\subsection{Applied technologies}

\subsubsection{Calibration}

Automatically performed by the operating system, calibration is an essential part of the measuring sequence. Prior to any measurement, the system must be calibrated for a particular impedance range with a known calibration resistor by calculating the system gain factor, see Table 1 and Figure 3 B. Two selectable precision resistors are integrated per headstage, as shown in Figure 2 A. High isolation reed relays are used for configuring calibration setting and disconnecting the IUT while calibration. Calibration resistor values should be selected application-specific to get most accurate measuring results. Furthermore, it is necessary to recalibrate the system after changing excitation voltage or TIA gain.

\subsubsection{Out-of-range detection}

Unknown IUT can have impedance values exceeding permissible measuring ranges. The out-of-range detection identifies low impedance values, if TIA voltage output exceeds one of two dynamic range thresholds $<0.2 \mathrm{~V}$ and $>4.8 \mathrm{~V}$. This would result in an incorrect analog-to-digital conversion in the signal processing stage in Figure $2 \mathbf{A}$, because the short peaks of the sinusoidal signal are cut off. If low impedance values are detected by the control unit, excitation voltage, TIA gain and calibration resistor are gradually reduced for a new measurement, considering the automated range selection combinations in Table 1. Impedance values below the lowest limit in Table 1 remain invalid and a comment is written into the impedance data. Out-of-range detection is outsourced to an Arduino MICRO microprocessor board due to timing and interrupt restrictions.

\subsubsection{Data analysis}

Data analysis is performed offline from stored impedance values, as illustrated in Figures $3 \mathbf{A}$. Impedance data are semicolon-separated stored in kiloohms $(\mathrm{k} \Omega)$ in a text file (*.CSV) on a microSD memory card, see Figure 1. After calibration, five impedance measuring points are sampled, used for online calculation of their mean and relative error. Each line of the $30 \mathrm{MB}$ delimited CSV file contains data records (mean and measuring points) from all headstages with corresponding time stamp and a short status report. Time stamps are generated by an integrated real-time clock with long-term battery backup. Our filtering strategy is based on the elimination of noise sources. Therefore, the estimated error is taken into account for data analysis as an index for measurement quality. Experiences have shown that even minor deviations indicate noise coupling due to electromagnetic fields. Relative errors that exceed the threshold value of $0.5 \%$ are indicated in the status report. The status report also documents successful measurements, out-of-range warnings and disconnected headstages.

\subsubsection{Shielding and grounding}

Special environments require special shielding and grounding strategies. We prefer the use of Faraday cages to block electromagnetic signals to the IUT and headstages. Therefore, the control unit is galvanically isolated to the headstages and all external control signals. In addition, the mixed-signal design of the analyzer is divided into analog and digital parts with separated supply voltages and floating grounds. The rear panel is equipped with several ground sockets allowing a flexible grounding concept for optimum SNR, including connections to potential earth and faraday cages.

\section{Results}

\subsection{Comparative measurements}

Comparative measurements with known IUT components (resistors, capacitors and mixed RC networks) were obtained for impedance analyzer accuracy verification. The analyzer was equipped as described in Table 1, measuring three IUT in parallel. Measuring sequences were started with a five minute interval timer. The high-accuracy impedance analyzer 
HP4194A (Hewlett Packard, USA) was used as reference to make a direct comparison of the determined impedance values. In addition, a Keithley 2000 multimeter (Keithley Instruments Inc., USA) features resistance measurements for real part estimation of the complex impedance. Table 2 lists impedance analyzer results as arithmetic mean together with reference measurements.

Table 2: Comparative impedance measurements

\begin{tabular}{|c|c|c|c|c|}
\hline \multirow{2}{*}{$\begin{array}{c}\text { IUT } \\
\text { resistor }\end{array}$} & \multicolumn{2}{|c|}{ reference value } & \multicolumn{2}{|c|}{ analyzer } \\
\hline & Keithley & HP4194A & mean & points \\
\hline $10 \mathrm{M} \Omega^{* *}$ & $9.99 \mathrm{M} \Omega$ & $9.98 \mathrm{M} \Omega$ & $9.97 \mathrm{M} \Omega$ & 240 \\
\hline $7.0 \mathrm{M} \Omega^{\star \star}$ & $6.99 \mathrm{M} \Omega$ & $7.04 \mathrm{M} \Omega$ & $6.95 \mathrm{M} \Omega$ & 240 \\
\hline $5.0 \mathrm{M} \Omega^{\star *}$ & $4.99 \mathrm{M} \Omega$ & $5.02 \mathrm{M} \Omega$ & $4.97 \mathrm{M} \Omega$ & 240 \\
\hline $1.5 \mathrm{M} \Omega$ * & $1.50 \mathrm{M} \Omega$ & $1.50 \mathrm{M} \Omega$ & $1.50 \mathrm{M} \Omega$ & 1080 \\
\hline $1.0 \mathrm{M} \Omega$ * & $1.00 \mathrm{M} \Omega$ & $0.99 \mathrm{M} \Omega$ & $1.00 \mathrm{M} \Omega$ & 1000 \\
\hline $200 \mathrm{k} \Omega^{\star *}$ & $199.6 \mathrm{k} \Omega$ & $199.3 \mathrm{k} \Omega$ & $200.4 \mathrm{k} \Omega$ & 1000 \\
\hline $68 \mathrm{k} \Omega^{\star \star}$ & $67.9 \mathrm{k} \Omega$ & $67.8 \mathrm{k} \Omega$ & $68.6 \mathrm{k} \Omega$ & 1080 \\
\hline capacitor & calculated & HP4194A & mean & points \\
\hline $100 \mathrm{pF}^{* \star *}$ & $1.59 \mathrm{M} \Omega$ & $1.59 \mathrm{M} \Omega$ & $1.54 \mathrm{M} \Omega$ & 1000 \\
\hline parallel RC & calculated & HP4194A & mean & points \\
\hline $10 \mathrm{M}^{* *} / / 100 \mathrm{pF}^{\star * *}$ & $1.57 \mathrm{M} \Omega$ & $1.61 \mathrm{M} \Omega$ & $1.61 \mathrm{M} \Omega$ & 1080 \\
\hline
\end{tabular}

\subsection{Long-term measurements}

To illustrate the accuracy and stability of analyzer measurements, the 20-hour graphs in Figure 4 represent the percentage deviation of the measured analyzer values in relation to the IUT reference values in Table 2, reduced to every third data point.

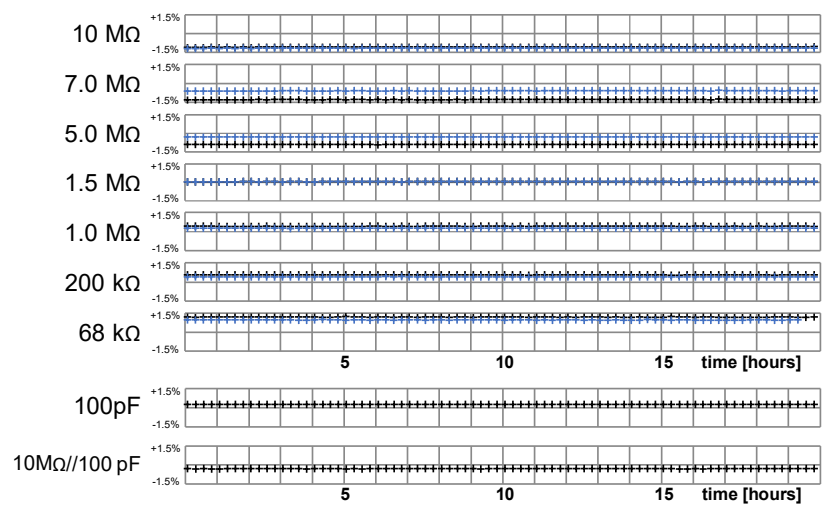

Figure 4: Percentage deviation of measured IUT related to reference values (blue: Keithley, black: HP4194A). The measured real parts (Keithley) of IUT $100 \mathrm{pF}$ and $10 \mathrm{M} \Omega / / 100 \mathrm{pF}$ are not considered. All data were reduced to every third data point.
It was found that the determined impedance values were measured stable and well within the tolerance limit of $\pm 1.5 \%$.

\section{Conclusion}

The presented multi-channel impedance analyzer offers highly accurate impedance measurements from $50 \mathrm{k} \Omega$ to $10 \mathrm{M} \Omega$ with an excitation frequency of $1 \mathrm{kHz}$. The analyzer was equipped as described in Table 1, currently optimized for application-specific IUT values near $0.5 \mathrm{M} \Omega$ and $2 \mathrm{M} \Omega$, due to calibration and TIA gain settings. With a system accuracy better than $1.5 \%$, the determined differences between values from analyzer and reference measurements were negligible, see Table 2. High repeatability of impedance analyzer measurements was reflected in small standard deviations of less than $1 \mathrm{k} \Omega$ for all presented results in Table 2 . Out-of-range detection is a key feature that was integrated to optimize measurement parameters and to ensure examination of unknown IUT in the permissible dynamic range. Incorrect measurements due to long-term drifts, offsets and self-heating are avoided by performing automated calibration before measuring. The presented impedance analyzer is a low cost system ready for use particularly in long-term characterization of dielectric material properties with multiple samples.

\section{Author Statement}

Research funding: This work was partially funded by the Federal Ministry of Education and Research (BMBF) within the project RESPONSE "Partnership for Innovation in Implant Technology". Conflict of interest: Authors state no conflict of interest. Informed consent is not applicable. Ethical approval: Not applicable.

\section{References}

[1] Data sheet 1 MSPS, 12-Bit Impedance Converter, Network Analyzer AD5933 Rev. F, Analog Devices, Inc., Norwood, MA, USA. 2017

[2] Evaluation Board User Guide UG-364 Rev.0, Analog Devices, Inc., Norwood, MA, USA. 2012

[3] Impedance Measurement, Handbook $6^{\text {th }}$ Edition, Application Note, Keysight Technologies, USA. 2016 